\title{
Book review: Wider dem europäischen Austeritätsregime: Mit Laclau, Mouffe, Rancière und Lefebvre das transformatorische Potenzial politischer Kämpfe ausschöpfen
}

\author{
Martina Blank \\ Institut für Humangeographie, Goethe-Universität Frankfurt, Theodor-W.-Adorno-Platz 6, \\ 60323 Frankfurt am Main, Germany \\ Correspondence: Martina Blank (mblank@em.uni-frankfurt.de)
}

Published: 11 April 2018

Mullis, D.: Krisenproteste in Athen und Frankfurt: Raumproduktionen der Politik zwischen Hegemonie und Moment, Münster, Westfälisches Dampfboot, 367 S., ISBN: 978-389691-299-2, EUR 36,00, 2017.

In seinem Buch Krisenproteste in Athen und Frankfurt. Raumproduktionen der Politik zwischen Hegemonie und Moment, das Ende des Jahres 2017 in der Reihe „Raumproduktionen“ im Verlag Westfälisches Dampfboot erschienen ist, geht Daniel Mullis den Fragen nach, wie und unter welchen Bedingungen emanzipatorische Akteure Veränderungen erkämpfen können. Mullis ist es daran gelegen, Hegemonie und Moment als zwei sich ergänzende Modi der Politik zusammenzubringen und ein relationales und prozessuales Verständnis der gegenseitigen Bedingtheit von Raum und Politik zu entwickeln. Dazu verbindet er die politischen Philosophien von Ernesto Laclau und Chantal Mouffe (2000) einerseits und von Jacques Rancière (2002) andererseits mit der Raumtheorie Henri Lefebvres (1991). Illustriert wird dies mit den Krisenprotesten in Athen zwischen 2008 und 2014 und den Aktivitäten des Blockupy-Bündnisses in Frankfurt am Main zwischen 2012 und 2015.

Das ursprünglich als Dissertation im Bereich Humangeographie entstandene Buch beinhaltet damit zwei sich ergänzende Projekte: Zum einen ist es Mullis' explizites Anliegen, durch sein Buch zur kollektiven Geschichtsschreibung der Krisenproteste beizutragen (S. 19). Die Fallstudien zu Frankfurt und Athen, die ausdrücklich nicht als Vergleich angelegt sind, können daher auch für sich gelesen werden. Auch wenn sich Mullis' Darstellung dabei teilweise überreichlich auf Daten, Zahlen und Fakten stützt, liest sich dieser empirische Teil in weiten Teilen spannend und lässt die Kraft der Proteste wieder auferstehen. Dabei orientiert sich die Beschreibung der Proteste, ihrer Entwicklung und Zielsetzungen, an dem Verhältnis von momenthaften Mobilisierungen einerseits und kontinuierlichen Kämpfen andererseits sowie der Rolle von Orten und Räumen für diese (S. 120-121).

Zum anderen handelt es sich bei dem Buch aber auch um ein anspruchsvolles theoretisches Projekt. Dafür führt Mullis zunächst das Konzept von Hegemonie in Anschluss an Laclau und Mouffe ein, um das Funktionieren und die Wirkungsweise kontinuierlicher Kämpfe und die über Verknüpfung vollzogene Verallgemeinerung politischer Projekte $\mathrm{zu}$ denken. Diesem Fokus auf kontinuierliche Auseinandersetzungen und verknüpfende Praxen stellt Mullis' Überlegungen Rancières zur Bedeutung der Singularitäten politischer Momente gegenüber, um explosive Mobilisierungen und spezifische Ereignisse als aufbrechende Momente zu konzeptionalisieren. Zur Vermittlung der beiden Ansätze rekurriert Mullis auf die von Oliver Marchart formulierte postfundamentalistische politische Ontologie (Marchart, 2013), um die „,durch Praxis vollzogene Konstitution von Welt in konflikthaften Auseinandersetzungen und Machtrelationen denken zu können“, ,die Produktion von Gesellschaft [...], die als Ordnung institutionalisiert wird, aber nie in Gänze stabilisiert werden kann“ (S. 33).

Dem zentralen Diktum kritischer geographischer Forschung folgend, dass Gesellschaft und Raum verschränkt zu betrachten sind, verknüpft Mullis im Weiteren die Überlegungen $\mathrm{zu}$ Hegemonie und Moment mit der Raumtheorie 
Lefebvres. Aus der Diskussion Lefebvres entwickelt Mullis die beiden Begriffspaare "Raumproduktion der Politik“ und „Politisierung durch Raumproduktionen“ um ein zutiefst praxisorientiertes Verständnis der wechselseitigen Verschränkungen von Raum und Politik zu entwickeln. Dabei geht es um die Rolle von Orten und Räumen für politische Mobilisierungen und die Bedeutungen, die diese ihnen zuweisen. „Raumproduktionen“, so Mullis, ,,sind zugleich Modus wie Ausgangspunkt von Politik“ (S. 298). In der Empirie fragt Mullis danach ,wie, warum und wo durch Politik Raum produziert wurde und welche Rolle verstetigte Raumproduktionen für Politik spielen“ (S. 297-298).

Mullis' Bemühung, die Konzepte von Laclau, Mouffe, Rancière und Lefebvre nicht nur in der Empirie zu verknüpfen, sondern ihre Verzahnung auch theoretisch zu plausibilisieren, macht den Text zwar in Teilen äußerst sperrig, bietet dafür aber auch manche theoretische Detailerkenntnis, die ihren eigenen Wert jenseits des Gesamtargumentationsgangs besitzt.

Ein Beispiel hierfür bietet Mullis' Auseinandersetzung mit der oft zitierten Triade der Raumproduktion Lefebvres. In der Literatur werden die beiden Triaden „Repräsentation des Raums“, „Räumliche Praxis“ und „Räume der Repräsentation“ einerseits sowie die Triade vom „konzipierten Raum“, „wahrgenommenen Raum“ und „gelebten Raum“ anderseits oftmals in eins gesetzt und die drei jeweiligen Pole auf derselben Abstraktionsebene angesiedelt (z.B. Ronneberger und Vogelpohl, 2014; Schmid, 2010). Mullis schlägt demgegenüber eine andere Lektüre Lefebvres vor, der zufolge die Repräsentationen des Raums und die Räume der Repräsentation als binäre Opposition zu denken sind, als einander gegenübergestellte Räume der Herrschenden und Räume der unterworfenen Nutzer*innen, die durch die auf einer grundsätzlicheren Ebene angesiedelte räumliche Praxis miteinander vermittelt sind. Die zweite Trias von konzipiertem, wahrgenommenem und gelebtem Raum bezeichnet dann wiederum verschiedene Aspekte der beiden einander gegenübergestellten Räume. In den Worten Mullis': „Naheliegender als die beiden Triaden als Deckungsgleich zu verstehen, ist die beiden konkreten Pole des Konflikts, die Repräsentationen des Raums sowie die Räume der Repräsentation durch sämtliche Aspekte der zweiten Triade zu fassen.“ (S. 82). Für diese Rekonzeptionalisierung der Lefebvreschen Trialektik spricht vor allem ihre verbesserte Nachvollziehbarkeit für empirische Studien und auch für politische räumliche Praxis. Leider bleibt die theoretische Auseinandersetzung mit Lefebvre, wie Mullis selbst anmerkt, dem Gesamtargumentationsgang aber in Teilen äußerlich, so dass die weitere Anwendung der theoretischen Innovation der Kreativität der Leser*innen überlassen bleibt.

Doch auch wenn die Fallstudie und die theoretische Arbeit bezogen auf die Produktion des Raums besser aufeinander bezogen sein könnten, gewinnt das theoretische Argument zur Verknüpfung von Hegemonie und Moment durchaus Plausibilität durch die Fälle. So ist das Spiel mit Hege- monie und Moment sehr nützlich, um die jeweilige Spezifik der Mobilisierung in Athen und Frankfurt weniger bezogen auf ihre unterschiedlichen Kontexte oder Bezüge, als vielmehr bezogen auf die Art, Politik zu machen, zu begreifen. Dadurch lässt sich gerade auch für die Praxis vieles über Möglichkeiten aber auch Grenzen von Mobilisierungsstrategien lernen. Eruptive Momente, wie die spontane, massenhafte Besetzung des Syntagma-Platzes in Athen bleiben kontingent. „Momente können weder organisiert noch verstetigt werden, sie brechen Bahn und leben von der Spontaneität und Kreativität, die sie freisetzen, wenn sie den Horizont des Möglichen erweitern“ (S. 306). Diese Erfahrung ließ sich in den vergangenen Jahren vielerorts beobachten - vom so genannten arabischen Frühling zu den Platzbesetzungen in Spanien, der Türkei u.v.m. Aber solche Momente gründen oftmals in vorangegangenen Praxen der Hegemonie und verschieben die Bedingungen für diese (S. 305). Praxen der Hegemonien ermöglichen, vertiefen und verbreitern Momente (S. 306). Besonders deutlich wird dies an den Beispielen des freien Theaters Embros in Athen und der langanhaltenden, Spektren übergreifenden Zusammenarbeit des Blockupy-Bündnisses. Dabei, so zeigt Mullis, geht es auch um Vertrauensarbeit, die Zeit und Raum braucht. Auch hier lassen sich Anschlüsse zu anderen Bewegungszyklen wie den globalisierungskritischen Bewegungen oder auch den mexikanischen Zapatist@s finden.

Kritisch anzumerken ist hier, dass Mullis wenig Bezug zu diesen früheren Zyklen und der Forschung zu diesen herstellt. So wäre für Athen und Blockupy vielleicht nicht nur von Laclau, Mouffe und Rancière zu lernen gewesen, sondern auch von den zahlreichen Studien zu früheren Protestbewegungen. Mullis' explizite Abgrenzung zur Bewegungsforschung, die auf knapp zwei Seiten abgehandelt wird (S. 3031), birgt auch das Risiko, Brüche zu überschätzen und Kontinuitäten zu übersehen. Hegemonie gründet eben auch im durch die Bewegungsforschung aufbereiteten kollektiven Bewegungswissen, wie z.B. die jüngste Reise der Asamblea als Organisationsprinzip von den zapatistischen Gemeinden durch die globalisierungskritische Bewegung nach Athen zeigt. So entfernt sich Mullis auch in seinen Fragen, die er an die Proteste richtet, von den bewegungspolitischen Debatten der vergangenen Jahrzehnte und verortet seinen Text eher in einer longue durée politischer Kämpfe und traditioneller Oppositionen politischer Strömungen, wie sie schon seit dem 19. Jahrhundert fortexistieren. Andererseits liegt vielleicht gerade hier die Stärke des Arguments, indem Mullis ein brauchbares Angebot formuliert, Protest und Institutionalisierung jenseits des in politischen Debatten immer wieder neu aufgelegten Gegensatzes von Reform vs. Revolution als gleichermaßen bedeutsame Elemente für Emanzipation neu zu denken. Schließlich schlägt Mullis damit auch eine Brücke zwischen deutschsprachiger Bewegungsforschung und englischsprachigen Debatten zur Verschränkung von Politik, Raum und Stadt (S. 18-19). 
Für wissenschaftliche Anschlüsse hätte noch eine konkretere Beschreibung der durchgeführten Methode interessiert. Mullis verweist auf die Situationsanalyse von Adele Clarke (2012), die sich für derartige Projekte tatsächlich anbietet. Clarke hat mit ihrer Situationsanalyse einen methodischen Vorschlag entwickelt, die Grounded Theory durch postmoderne Forschungsperspektiven zu erweitern und so einerseits die Analyse diskursiver und materieller Praktiken zu kombinieren und andererseits totalisierende Konzepte von Gesellschaft oder ihren Teilen durch das offenere Konzept der Situation zu ersetzen. Das macht für Mullis' Projekt sehr viel Sinn. Die Verzahnung mit den theoretischen Überlegungen zu Laclau/Mouffe, Rancière und Lefebvre sowie ihrer konkreten Umsetzung wäre daher sehr aufschlussreich gewesen und hätte ähnliche Projekte anleiten können. Es bleibt zu wünschen, dass Mullis hierzu noch einmal gesondert nachlegt.

\section{Literatur}

Clarke, A. E.: Situationsanalyse: Grounded Theory nach dem Postmodern Turn, Wiesbaden, Springer VS, 2012.

Laclau, E. und Mouffe, C.: Hegemonie und radikale Demokratie: Zur Dekonstruktion des Marxismus, Wien, Passagen, 2000.

Lefebvre, H.: The Production of Space, Oxford, Blackwell, 1991.

Marchart, O.: Das unmögliche Objekt: Eine postfundamentalistische Theorie der Gesellschaft, Berlin, Suhrkamp, 2013.

Rancière, J.: Das Unvernehmen: Politik und Philosophie, Frankfurt a.M., Suhrkamp, 2002.

Ronneberger, K. und Vogelpohl, A.: Henri Lefebvre: Die Produktion des Raumes und die Urbanisierung der Gesellschaft, in: Theorien in der Raum- und Stadtforschung: Einführungen, Herausgeber: Oßenbrügge, J. und Vogelpohl, A., Münster, Westfälisches Dampfboot, 251-270, 2014.

Schmid, C.: Stadt, Raum und Gesellschaft: Henri Lefebvre und die Theorie der Produktion des Raumes, 2. Aufl. Stuttgart, Franz Steiner Verlag, 2010. 